\title{
The K2 RR Lyrae Survey
}

\author{
Róbert Szabó ${ }^{1,3, \star}$, Katrien Kolenberg ${ }^{2,3,4}$, László Molnár ${ }^{1}$, Emese Plachy ${ }^{1}$, Péter Klagyivik ${ }^{1}$, Ottó Hanyecz ${ }^{1,5}$, and \\ the KASC RR Lyrae and Cepheid Working Group members \\ ${ }^{1}$ Konkoly Observatory, Research Centre for Astronomy and Earth Sciences, Hungarian Academy of Sciences, H-1121 Budapest, \\ Konkoly Thege Miklós út 15-17, Hungary \\ ${ }^{2}$ Harvard-Smithsonian Center for Astrophysics, 60 Garden Street, Cambridge MA 02138, USA \\ ${ }^{3}$ Instituut voor Sterrenkunde, KU Leuven, Celestijnenlaan 200D, 3001 Heverlee, Belgium \\ ${ }^{4}$ Department of Physics, University of Antwerp, Groenenborgerlaan 171, 2020 Antwerp, Belgium \\ ${ }^{5}$ Eötvös Loránd Tudományegyetem, H-1117 Pázmány Péter sétány 1/A, Budapest, Hungary
}

\begin{abstract}
We have initiated a large survey with K2 to observe thousands of RR Lyrae stars along the Ecliptic. The high photometric precision and the 80-90-day continuous coverage will allow us to investigate the light variation of these galactic structure tracer variable stars with an unprecedented detail. The survey will help us to conduct a thorough statistical study of RR Lyrae pulsation dynamics including old and recently discovered dynamical phenomena, like resonances, non-radial modes, period doubling, and the Blazhko effect. We describe the survey, present the first results and discuss the prospects of combining the K2 RR Lyrae survey, Gaia, and LSST in the context of galactic structure studies.
\end{abstract}

\section{Introduction}

With the failure of the second reaction wheel of Kepler, a new mission, dubbed K2 was conceived [1]. The new mission observes ecliptic fields for 80-90 days with a precision which is comparable to the original mission. $\mathrm{K} 2$ is a bona fide community endeavour: without a central, pre-determined research plan, exoplanets, stellar (asteroseismic and other variable) sources, extragalactic objects, microlensing events, and even Solar System objects have been proposed by community members. This opened up new possibilities in RR Lyrae research, too, since compared to the original Kepler field, where roughly 50 RR Lyrae stars were found, hundreds or even thousands of RR Lyrae stars can be observed in K2. Members of the Kepler Asteroseismic Science Consortium (KASC) RR Lyrae and Cepheids Working Group initiatied a large survey to ensure that as many RR Lyrae stars will be observed with $\mathrm{K} 2$, as possible. This contribution discusses the present status of this undertaking.

\section{The K2 RR Lyrae survey}

The project has been very successful in proposing and observing RR Lyrae stars with K2, see figure 1 for a graphical representation of observed an planned RR Lyrae stars along the Ecliptic, while figure 2 demonstrates the number of approved/observed RR Lyrae targets [2] and also the success rate of each submitted proposal up to the submission of this contribution. Very briefly we can say that

\footnotetext{
^e-mail: szabo.robert@csfk.mta.hu
}

apart from some instrumental problems (shift in the fieldof-view, high contamination in the Galactic Bulge) almost all our proposed targets have been accepted and observed. The total number of RR Lyrae targets is over 3400 up to Campaign 13.

The sample covers RR Lyrae in extragalaxies (Sagittarius dwarf galaxy, Leo IV [3]), globular clusters (M80, M4), the Galactic Bulge (Campaign 9), and several Galactic fields along the Ecliptic both in high and low galactic latitudes, the Galactic center and anticenter directions, halo and field stars, as well as prominent halo structures, such as the Sagittarius stream.

The advantage of the K2 RR Lyrae survey is at least threefold. First, it will be a statistical mission. The project will allow us to study the occurrence rate and statistics of newly found dynamical phenomena, like period doubling, resonances, chaos, modulations, nonradial modes and their correlation with age, metallicity, and galactic position. Besides the statistics factor, this extensive survey will also provide rare gems, such as (classical) doublemode stars, stars with anomalous period ratios, rare types of modulation, modulated RRc stars, and so on. Thirdly, the high quality light curves will provide a golden sample to calibrate and test the algorithms and performance of and exploit synergies with upcoming large sky surveys, like Gaia [4] and the Large Synoptic Survey Telescope (LSST) [5]. 


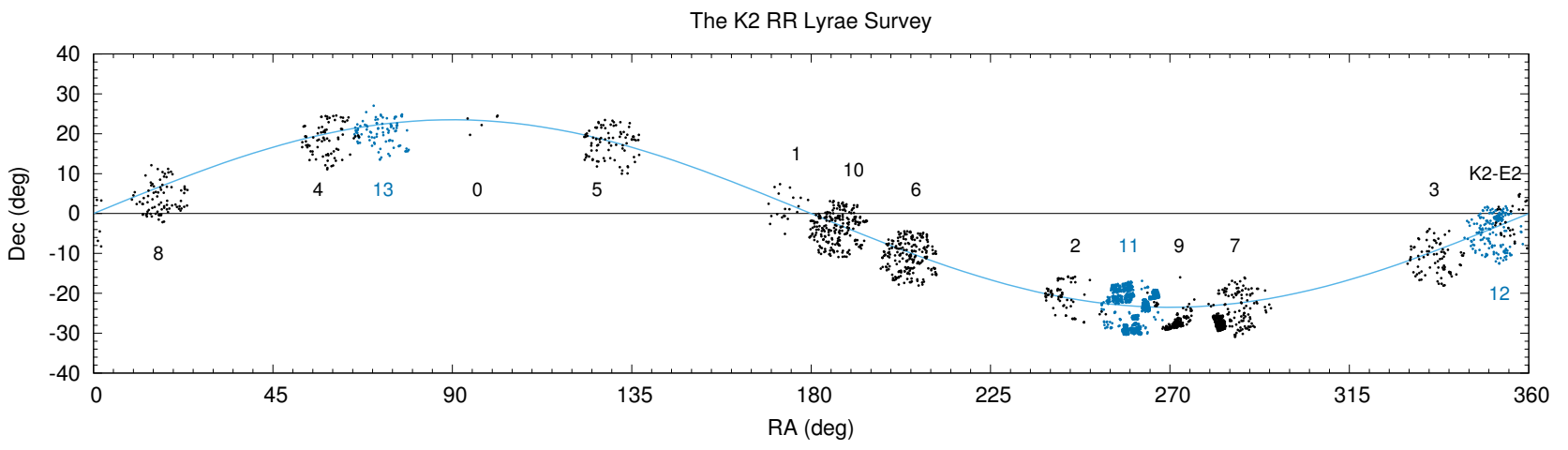

Figure 1: The footprint of the K2 RR Lyrae survey on the sky. Approved (blue) and observed (black) RR Lyrae targets are marked in the $\mathrm{K} 2$ campaign fields.

\section{Preliminary results}

While the K2 RR Lyrae survey is ongoing, some results have already been published or will be published soon. Here we summarize the most important highlights so far:

1. We expect to observe a sizeable sample of RRd stars, that were not present in the original Kepler field, and so far have been observed only with MOST [6] and CoRoT [7].

2. During the 8.9-d long K2 Two-Wheel Concept Engineering Test 33 RR Lyrae stars have been observed [8]. Besides fundamental-mode (RRab) and first overtone (RRc) pulsators, the sample includes the first two doublemode (RRd) stars that Kepler detected. All RRd and nonmodulated RRc stars show the additional mode with a period ratio of 0.61 with the first overtone radial mode. As a by-product of the target search and analysis, we identified 165 bona fide double-mode RR Lyrae stars from the Catalina Sky Survey observations throughout the sky, 130 of which are new discoveries.

3. We also discovered a peculiar double-mode (RRd) star, both modes of which are modulated in antiphase. This is hitherto the only modulated RRd star that can be studied with accurate space-borne photometry (Plachy et al. in this proceedings). The results of a detailed investigations will be published in a dedicated paper (Plachy et al. in prep).

4. A strongly modulated (Blazhko) RRc star was found [8]. This is the only modulated first-overtone RR Lyrae ever observed from space, providing a unique window on the mechanism of the Blazhko-modulation in RRc stars.

5. We presented the first observations of extragalactic pulsating stars in the $\mathrm{K} 2$ ecliptic survey of the Kepler space telescope. The dwarf spheroidal galaxy Leo IV contains three known RR Lyrae stars. All three were observed by $\mathrm{K} 2$ during Campaign 1 . These objects are among the faintest targets ( $\mathrm{Kp} 21.5 \mathrm{mag}$, but see also [9]) the Kepler/K2 missions have observed. We identified one modulated star and another likely Blazhko candidate with periods of $29.8+-0.9 \mathrm{~d}$ and more than $80 \mathrm{~d}$, respectively. Therefore we unambiguously detected Blazhko modulation in an extragalaxy beyond the Magellanic Clouds [3]. Two out of three stars are blends with brighter background galaxies in the $\mathrm{K} 2$ frames, hence image subtraction was a vital part of the analysis, and will be for future space photometric missions with large pixel sizes (e.g. TESS [10], PLATO [11]), as well.

6. Thanks to high-precision, continuous space-borne as well as ground-based photometry the Petersen-diagram of RR Lyrae stars has started to be populated with exotic and new types of periodicities (see e.g. Molnár et al. in these proceedings). Besides the half-integer frequencies due to period-doubling [12], extra frequencies have been found around the expected location of the second radial overtone frequency [13], around $P_{\mathrm{X}} / P_{1}=0.61$ [14], where $P_{1}$ and $P_{\mathrm{X}}$ are the periods of first radial overtone and a presumably nonradial mode which seems to be ubiquitous in RRc and RRd stars [15]. In addition, anomalous first overtone / fundamental mode $\left(P_{1} / P_{0}\right)$ period ratios were uncovered [16], [17], [18], [19].

All these additional frequencies that have been unveiled in recent years can be studied in exquisite details within the K2 RR Lyrae survey.

\section{Conclusions}

We introduced the K2 RR Lyrae survey, which will provide excellent means to study the pulsation mechanism and dynamical phenomena of these old, horizontal-branch objects, as well as to conduct galactic structure studies, since many of our targets trace different (extra)galactic components, like globular clusters, halo streams, and dwarf galaxies. The synergies with Gaia and LSST are numerous. Gaia will observe parallaxes and hence luminosities, but the temporal photometric coverage will be inferior compared to K2. LSST will go much deeper and will see much fainter objects, but the sampling rate will not allow the investigation of detailed temporal behavior of the pulsation. Combining the results of these surveys with the K2 sample will offer a unique opportunity to study RR Lyrae stars with a holistic approach. TESS and PLATO will continue and extend that work beyond the Ecliptic fields after $\mathrm{K} 2$ comes to an end.

We note that it is not trivial to derive the clean $\mathrm{K} 2$ light curves of large-amplitude variables (free of instrumental 


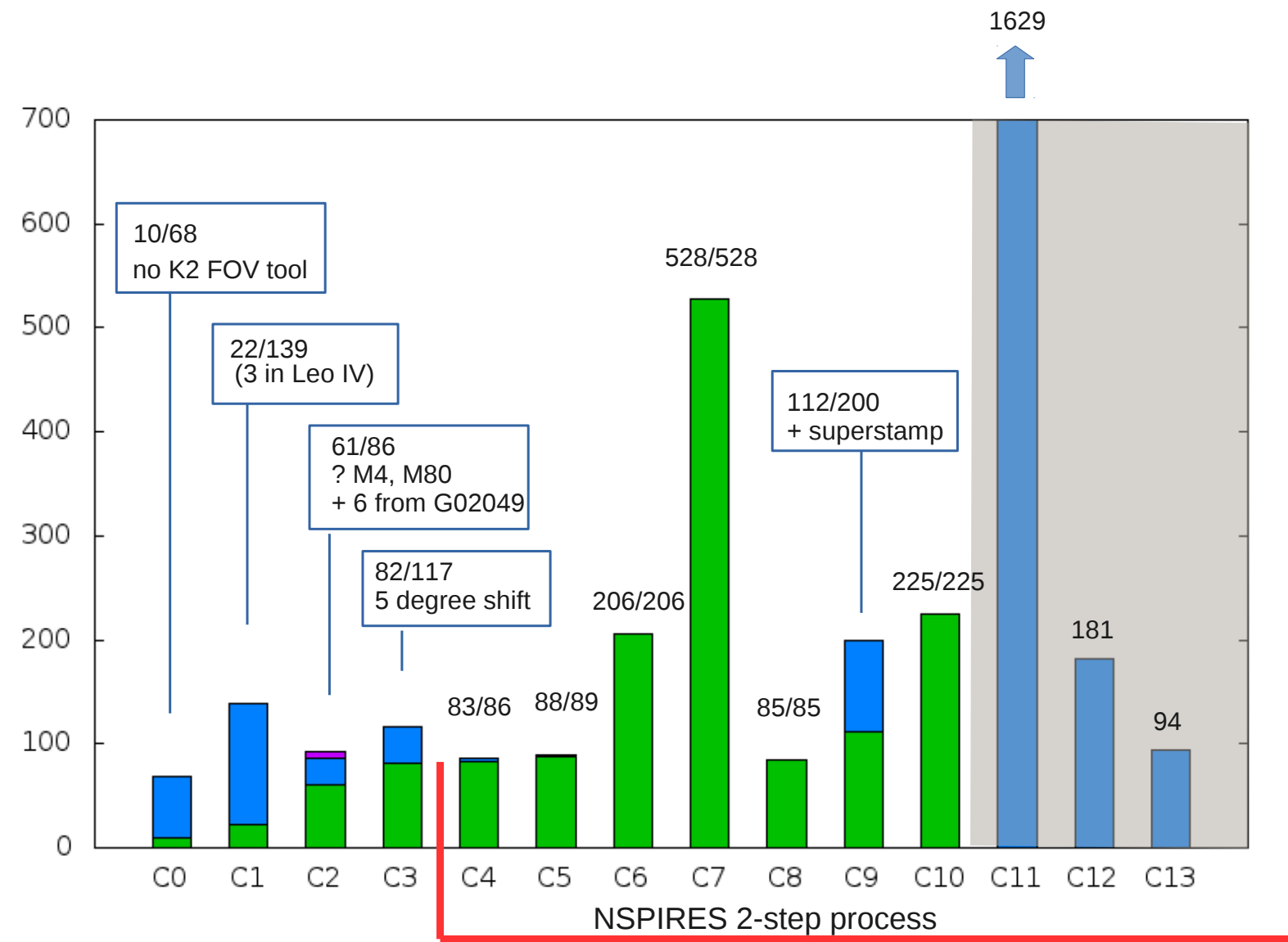

Figure 2: Statistics of the number of proposed (blue) and observed (green) RR Lyrae targets in the K2 campaigns. The K2 RR Lyrae survey has been largely successful in securing most of the known RR Lyrae stars for K2 observations.

effects), see e.g. the contribution of Plachy et al. on the EAP photometric method in these proceedings. Most of the published algorithms are optimized for transit search or the detection of low-amplitude solar-like oscillations. As a service to the community, the corrected K2 RR Lyrae light curves will be publicly available.

\section{Acknowledgements}

This project has been supported by the Lendület2009, LP201231, and LP2014-17 Young Researchers Programs of the Hungarian Academy of Sciences and by the NKFIH K115709, PD116175, and PD121203 grants of the Hungarian National Research, Development and Innovation Office. The research leading to these results has received funding from the European Community's Seventh Framework Programme (FP7/2007-2013) under grant agreement no. 312844 (SPACEINN), and the ESA PECS Contract No. 4000110889/14/NL/NDe. L. Molnár was supported by the János Bolyai Research Scholarship of the Hungarian Academy of Sciences.

\section{References}

[1] Howell, S., Sobeck, C., Haas, M. et al., PASP 126, 398, (2014)
[2] Plachy, E., Molnár, L., Szabó, R., Kolenberg, K., Bányai, E., CoKon 105, 19, (2016)

[3] Molnár, L., Pál, A., Plachy, E. et al., ApJ 812, 2, (2015a)

[4] Gaia Collaboration A\&A, in press, arXiv:1609.04153, (2016)

[5] Ivezić, Ž., Tyson, J. A., Abel, B. et al. arXiv:0805.2366, (2008)

[6] Gruberbauer, M., Kolenberg, K., Rowe, J. F. et al., MNRAS 379, 1498, (2007)

[7] Chadid, M., A\&A 540, 68 (2012)

[8] Molnár, L., Szabó, R., Szabó, Gy. M. et al., MNRAS 452, 4283, (2015b)

[9] Pál, A., Szabó, R., Szabó, Gy. M. et al., ApJL 804, L45, (2015)

[10] Ricker, G. R., Winn, J., Vanderspek, R. et al., Journal of Astronomical Telescopes, Instruments, and Systems 1, id. 014003 (2015)

[11] Rauer, H., Catala, C., Aerts, C. et al: ExpAstr 38, 249, (2014)

[12] Szabó, R., Kolláth, Z., Molnár, L. et al., MNRAS 409, 1244, (2010) 
[13] Benkő, J. M., Kolenberg, K., Szabó, R. et al., MNRAS 409, 1585, (2010)

[14] Moskalik, P., Smolec, R., Kolenberg, K. et al., MNRAS 447, 2348, (2015)

[15] Szabó, R., Benkő, J. M., Paparó, M. et al., A\&A 570, A100, (2014)

[16] Molnár, L., Kolláth, Z., Szabó, R. et al., ApJL 757, L13, (2012)
[17] Soszyński, I., Smolec, R., Dziembowski, W. A. et al., MNRAS 463, 1332, (2016)

[18] Jurcsik, J., Smitola, P., Hajdu, G., Nuspl, J. ApJ 797, $3,(2014)$

[19] Jurcsik, J., Smitola, P., Hajdu, G. et al., ApJS 219, 25, (2015) 